\title{
Correction: Marino, M.; Misuri, L.; Carati, A.; Brogioli, D. Proof-of-Concept of a Zinc-Silver Battery for the Extraction of Energy from a Concentration Difference. Energies 2014, 7, $3664-3683$
}

\section{Massimo Marino ${ }^{1}$, Lorenza Misuri ${ }^{1}$, Andrea Carati ${ }^{2}$ and Doriano Brogioli ${ }^{1, *}$}

1 Dipartimento di Scienze della Salute, Università degli Studi di Milano-Bicocca, via Cadore 48, 20900 Monza, Italy; E-Mails: massimo.marino@unimib.it (M.M.); lorenza.misuri@unimib.it (L.M.)

2 Dipartimento di Matematica, Università degli Studi di Milano, via Saldini 50, 20133 Milano, Italy; E-Mail: andrea.carati@unimi.it

* Author to whom correspondence should be addressed; E-Mail: dbrogioli@gmail.com; Tel.: +39-02-6448-8244.

We would like to change the authors' affiliations on Page 3664 of paper [1] from:

1 Dipartimento di Matematica, Università degli Studi di Milano, via Saldini 50, 20133 Milano, Italy; E-Mails: massimo.marino@unimib.it (M.M.); lorenza.misuri@unimib.it (L.M.)

2 Dipartimento di Scienze della Salute, Università degli Studi di Milano-Bicocca, via Cadore 48, 20900 Monza, Italy; E-Mail: andrea.carati@unimi.it

to the correct version as follows:

1 Dipartimento di Scienze della Salute, Università degli Studi di Milano-Bicocca, via Cadore 48, 20900 Monza, Italy; E-Mails: massimo.marino@unimib.it (M.M.); lorenza.misuri@unimib.it (L.M.)

2 Dipartimento di Matematica, Università degli Studi di Milano, via Saldini 50, 20133 Milano, Italy; E-Mail: andrea.carati@unimi.it

We apologize for any inconvenience caused to the readers. 


\section{Reference}

1. Marino, M.; Misuri, L.; Carati, A.; Brogioli, D. Proof-of-concept of a zinc-silver battery for the extraction of energy from a concentration difference. Energies 2014, 7, 3664-3683.

(C) 2014 by the authors; licensee MDPI, Basel, Switzerland. This article is an open access article distributed under the terms and conditions of the Creative Commons Attribution license (http://creativecommons.org/licenses/by/3.0/). 